Copyright (C 2012 IEEE. Personal use of this material is permitted. Permission from IEEE must be obtained for all other uses, in any current or future media, including reprinting/republishing this material for advertising or promotional purposes, creating new collective works, for resale or redistribution to servers or lists, or reuse of any copyrighted component of this work in other works. 


\title{
Excess Power Circulation in Distribution Networks Containing Distributed Energy Resources
}

\author{
Sumit Mazumder, Student Member, IEEE, Arindam Ghosh, Fellow, IEEE, Farhad Shahnia, \\ Firuz Zare, Senior Member, IEEE and Gerard Ledwich, Senior Member, IEEE
}

\begin{abstract}
The presence of large number of single-phase distributed energy resources (DERs) can cause severe power quality problems in distribution networks. The DERs can be installed in random locations. This may cause the generation in a particular phase exceeds the load demand in that phase. Therefore the excess power in that phase will be fed back to the transmission network. To avoid this problem, the paper proposes the use of distribution static compensator (DSTATCOM) that needs to be connected at the first bus following a substation. When operated properly, the DSTATCOM can facilitate a set of balanced current flow from the substation, even when excess power is generated by DERs. The proposals are validated through extensive digital computer simulation studies using PSCAD and MATLAB.
\end{abstract}

Keywords: DSTATCOM, DVR, DER, power circulation

\section{INTRODUCTION}

$\mathrm{D}$ UE TO the depletion in the traditional energy resource and the negative impact on environment of fossil fuels, renewable energy based generation is gaining substantial attention these days [1-3]. A distributed generator (DG) is usually installed in low voltage distribution networks at customer premises. Solar photovoltaic (PV) is the most popular form of DG, especially in countries with abundance of sunlight. In some countries, the installation of rooftop PVs by householders is accelerated due to government subsidy. The other forms of DG may include micro wind turbines, fuel cells, microturbines etc. Additionally, a large number of electric vehicles will be introduced in markets soon. Initially, they will only charge from the grid in G2V mode. However, as the battery technology improves, these can also supply power to the grid in V2G mode. Also storage technology (e.g., battery) will be used to supplement the variability of the some of the renewable sources. All these generation sources are collectively called distributed energy resources (DERs).

Due to the proximity of DERs to loads, transmission expense due to power loss can be reduced. However, introduction of DERs in LV networks has some drawbacks that need to be addressed. Some of the drawbacks are power quality problems like voltage and current unbalance, over and under voltage. Furthermore most of the LV distribution systems are radial in nature. All the protection and operational schemes are based on this nature. However, when DERs are connected to LV networks, they do not remain radial any more. This causes

The authors thank the Australian Research Council (ARC) for the financial support for this project through the ARC Discovery Grants DP 0774092 and DP110104554.

The authors are with Queensland University of Technology, Brisbane, Australia. protection issues like bidirectional power flow requiring changes in the overcurrent relay settings, sympathetic tripping and increased fuse blowing $[2,4]$.

The most frequent problem in LV distribution network is current and voltage imbalance [5]. Although the utility supply balanced voltage to the network, unequal distribution of single phase loads may create some unbalance in the LV side. Most of the DERs are single-phase and can have different ratings. Moreover, these will be deployed in LV networks at random locations. Therefore the net effect of these installations will ultimately affect the supply side since large unbalanced current be drawn from the MV substation [6].

Usually custom power devices are installed to alleviate the power quality problems in a distribution network [5]. A distribution static compensator (DSTATCOM) can balance the supply side currents, improve power factor by reactive power injection and control the voltage at a bus. It can also act as an active filter to suppress harmonic current drawn [7-8]. A dynamic voltage restorer (DVR), on the other hand, can supply a balanced voltage to a sensitive load by series voltage injection [5]. Some of these problems like harmonics, power loss have already investigated in the context of DERs to some extent [911]. It has been shown in [12] that a DSTATCOM can reduce voltage unbalance in a distribution network and the best possible location for installing DSTATCOM has also been investigated.

Since the DERs will be placed randomly, it may so happen that the generation in a particular phase exceeds the load demand in that phase. In such an event the excess power will be fed back in the substation and this will create severe current imbalance. To overcome this problem, a DSTATCOM is utilized in this paper. It is shown that the DSTATCOM has an ability to circulate extra generated phase power by the DERs among the phases of the system. To facilitate this, the DSTATCOM needs to be connected at the very first of the network. A particular circuit topology of the DSTATCOM is chosen that enables the circulation of power. Also the DSTATCOM current references are generated such that a set of balanced currents are drawn from the MV substation. The proposals are validated through extensive digital computer simulation studies using PSCAD and some load flow solutions using MATLAB.

\section{DSTATCOM STRUCTURE AND CONTROL}

The structure of the VSC realizing the DSTATCOM is shown in Fig. 1. It contains three H-bridges that are supplied from the common dc bus. The output of each H-bridge is connected to a T-filter consisting of an inductor $\left(L_{f}\right)$, a capacitor $\left(C_{f}\right)$ and the primary side of a single-phase transformer. The secondary sides of the transformers are connected in wye. The 
transformers provide galvanic isolation and voltage boosting. The turns ratio of each transformer is assumed to be $1: n$. The resistance $R_{f}$ represents the switching and transformer losses, while the inductance $L_{T}$ represents the leakage reactance of the transformers. The DSTATCOM will be controlled in output feedback current control mode using poles shift control [13], which is discussed below.

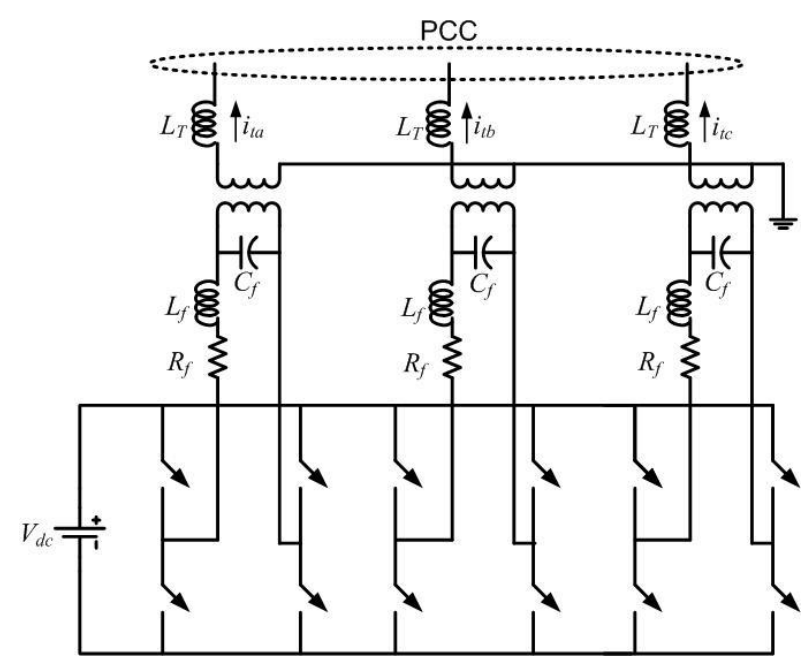

Fig. 1. Converter structure.

Let us consider only one phase of the DSTATCOM circuit, in which the following state vector is defined

$$
x^{T}=\left[\begin{array}{lll}
i_{t} & i_{f} & n v_{c f}
\end{array}\right]
$$

where $i_{t}$ is the injected current, $i_{f}$ is current flowing through the inductor $L_{f}$ and $v_{c f}$ is the voltage across the filter capacitor $C_{f}$. Then the state space equation of the system can be written as

$$
\begin{aligned}
& \dot{x}=A x+B z \\
& y=C x
\end{aligned}
$$

where

$$
\begin{aligned}
& A=\left[\begin{array}{ccc}
0 & 0 & \frac{1}{L_{T}} \\
0 & -\frac{R_{f}}{L_{f}} & -\frac{1}{n^{2} L_{f}} \\
-\frac{n^{2}}{C_{f}} & \frac{n^{2}}{C_{f}} & 0
\end{array}\right], \quad B=\left[\begin{array}{cc}
0 & -\frac{1}{L_{T}} \\
\frac{V_{d c}}{n L_{f}} & 0 \\
0 & 0
\end{array}\right] \\
& C=\left[\begin{array}{lll}
1 & 0 & 0
\end{array}\right], \quad z^{T}=\left[\begin{array}{ll}
u_{c} & v_{t}
\end{array}\right]
\end{aligned}
$$

where $u_{c}$ is the control law from which the switching will be generated and $v_{t}$ is the PCC voltage.

Assuming the PCC voltage as a disturbance input, the state equation (1) is converted into a discrete-time input-output equation as

$$
A\left(z^{-1}\right) y(k)=B\left(z^{-1}\right) u_{c}(k)
$$

where $A$ and $B$ are polynomials given by

$$
\begin{aligned}
& A\left(z^{-1}\right)=1+a_{1} z^{-1}+a_{2} z^{-2}+a_{3} z^{-3} \\
& B\left(z^{-1}\right)=b_{1} z^{-1}+b_{2} z^{-2}+b_{3} z^{-3}
\end{aligned}
$$

In the pole-shift control, the closed loop poles are obtained by radially shifting the open loop poles to a more stable location. The closed loop poles are then given by the polynomial

$$
T\left(z^{-1}\right)=A\left(\lambda z^{-1}\right)=1+a_{1} \lambda z^{-1}+a_{2} \lambda^{2} z^{-2}+a_{3} \lambda^{3} z^{-3}
$$

for $0<\lambda<1, \lambda$ being a scalar. The control law is then

$$
u_{c}(k)=\frac{S\left(z^{-1}\right)}{R\left(z^{-1}\right)}\left\{y_{r e f}(k)-y(k)\right\}
$$

The polynomials $R$ and $S$ are obtained from the solution of the equation

$$
T\left(z^{-1}\right)=A\left(z^{-1}\right) R\left(z^{-1}\right)+B\left(z^{-1}\right) S\left(z^{-1}\right)
$$

Based on this control law, the switching actions are taken as

$$
\begin{aligned}
& \text { If } u_{c}>h \text { then } u=+1 \\
& \text { elseif } u_{c}<-h \text { then } u=-1
\end{aligned}
$$

where $h$ is a small number.

\section{Distribution SySTEM COMPENSATION}

The DSTATCOM needs to circulate excess power generated by a phase to the remaining phase such that a set of balanced currents are drawn from the source. For this purpose, this device needs to be connected at the very first node following the substation. Moreover, if a single DSTATCOM can balance the source currents, no additional DSTATCOM will be required.

The distribution system under consideration is shown in Fig. 2, in which $v_{s}$ indicates the utility supply. The feeder impedance is denoted by $R_{s}$ and $L_{s}$. The source and load currents are denoted by $i_{s}$ and $i_{l}$ respectively, while the DSTATCOM injects a current $i_{t}$. We first assume that the DER is not connected to the system (switch $S$ is open).

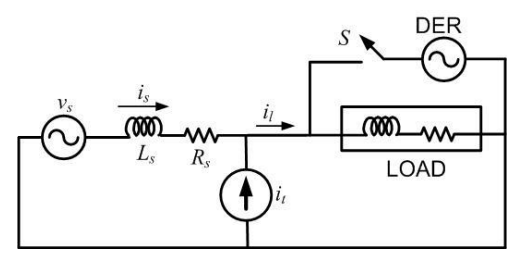

Fig. 2. Distribution system with DER and DSTATCOM.

For distribution system compensation, we shall use the theory of instantaneous symmetrical component [7]. The main aim here is to balance the source currents and make then in phase with the PCC voltages (Unity power factor). To apply the theory, first the fundamental positive sequence of the PCC voltage has to be obtained [14]. Let the instantaneous positive sequence voltages be denoted by $v_{t a}, v_{t b}$ and $v_{t c}$. The reference currents are given by

$$
\begin{aligned}
& i_{t a}^{*}=i_{l a}-\frac{v_{t a}}{M} P_{l a v} \\
& i_{t b}^{*}=i_{l b}-\frac{v_{t b}}{M} P_{l a v} \\
& i_{t c}^{*}=i_{l c}-\frac{v_{t c}}{M} P_{l a v}
\end{aligned}
$$


where

$$
M=\sum_{i=a, b, c} v_{t i}^{2}
$$

The quantity $P_{l a v}$ is the average load power, which is obtained by the average of the instantaneous load power given by

$$
p_{l}=v_{t a} i_{l a}+v_{t b} i_{l b}+v_{t c} i_{l c}
$$

It can be seen from Fig. 2 that the $i_{l}$ is the current supplied to the load minus the current injected by the DER when the switch $S$ is closed. Therefore the formulation (7) is general and will work even when the net power injected by the DERs is negative.

The current references generated through (7) will now be tracked using the output feedback control given in the previous section. Some numerical examples are now presented, the system parameters for these are given in Table I.

\section{TABLE-I: SYSTEM PARAMETERS}

\begin{tabular}{|l|l|}
\hline \multicolumn{1}{|c|}{ System Quantities } & \multicolumn{1}{c|}{ Values } \\
\hline Systems frequency & $50 \mathrm{~Hz}$ \\
\hline Source voltage & $11 \mathrm{kV}(\mathrm{L}-\mathrm{L}, \mathrm{rms})$ \\
\hline Feeder impedance & $R_{s}=3.025 \Omega, L_{s}=57.8 \mathrm{mH}$ \\
\hline Load impedance & Phase-a: $100+j 157.1 \Omega$ \\
& Phase-b: $150+j 235.62 \Omega$ \\
& Phase-c: $200+j 157.1 \Omega$ \\
\hline DSTATCOM & $R_{f}=9.68 \mathrm{~m} \Omega$ \\
parameters & $L_{f}=61.625 \mu \mathrm{H}$ \\
& $C_{f}=2348.8 \mu \mathrm{F}$ \\
& $V_{d c}=1.5 \mathrm{kV}$ \\
& Transformer: 1 MVA, $n=25$, \\
& $10 \%$ leakage \\
\hline
\end{tabular}

Example 1: In this example, we that the DER is connected to phase-a only at $0.2 \mathrm{~s}$ and the DSTATCOM is connected at $0.8 \mathrm{~s}$ in the network of Fig. 2. The DER is injects $150 \mathrm{~kW}$ and $5 \mathrm{kVar}$. The source currents before the DSTATCOM connection are shown in Fig. 3. It can be seen that they are unbalanced and the angle difference between the phases $a$ and $b$ is much less than $120^{\circ}$. The power drawn from the source by each phase before and after DER connection is shown in Fig. 4. It can be seen that phase-a was drawing $100 \mathrm{~kW}$ power before the DER connection, which then becomes negative 50 $\mathrm{kW}$ once the DER injects $150 \mathrm{~kW}$ power.

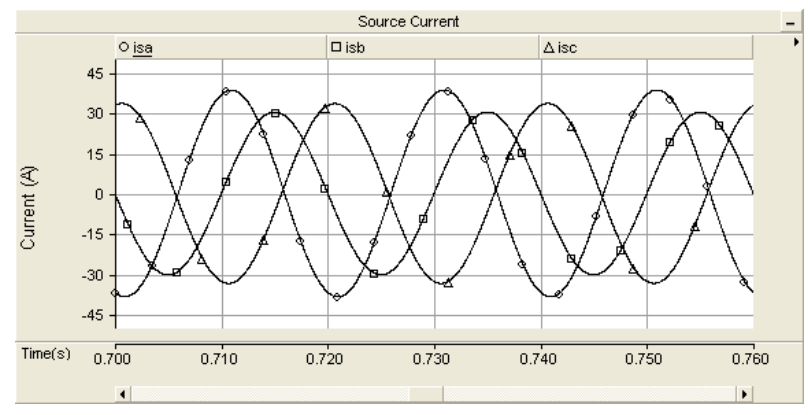

Fig. 3. Source current after DER connection (Example-1).

The results with when the DSTATCOM is connected at 0.8 $s$ are shown in Figs. 5 to 8. Fig. 5 shows the load currents, which are balanced. Since the excess power is now distributed in the other two phases, the source current magnitude has reduced. The source voltage before and after DSTATCOM con- nection is shown in Fig. 6. It can be seen that the voltage magnitude has increased slightly (about 5.5\%).

The power supplied by each phase of the source is shown in Fig. 7. It can be seen that they become equal as the DSTATCOM is connected indicating that the DSTATCOM is circulating power between the phases. The tracking performance is shown in Fig. 8, which shows both the reference and actual currents before and after DSTATCOM connection. It can be seen that the current tracking is very accurate.

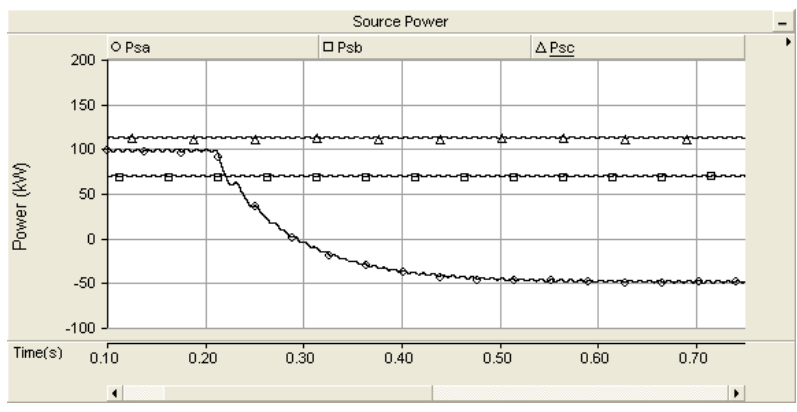

Fig. 4. Source power before and after DER connection (Example-1).

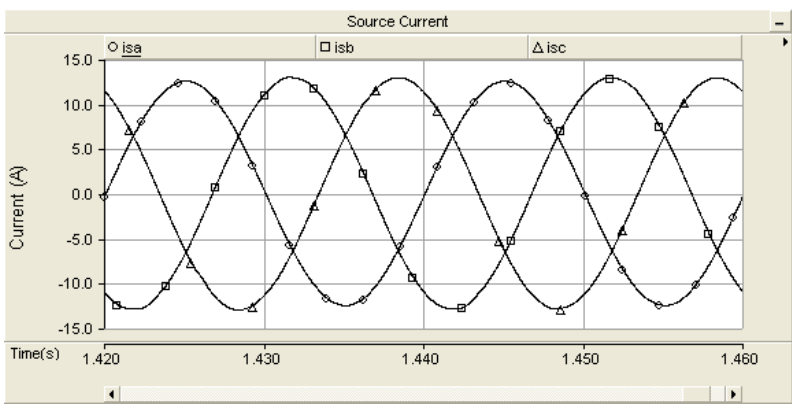

Fig. 5. Source current after DSTATCOM connection (Example-1).

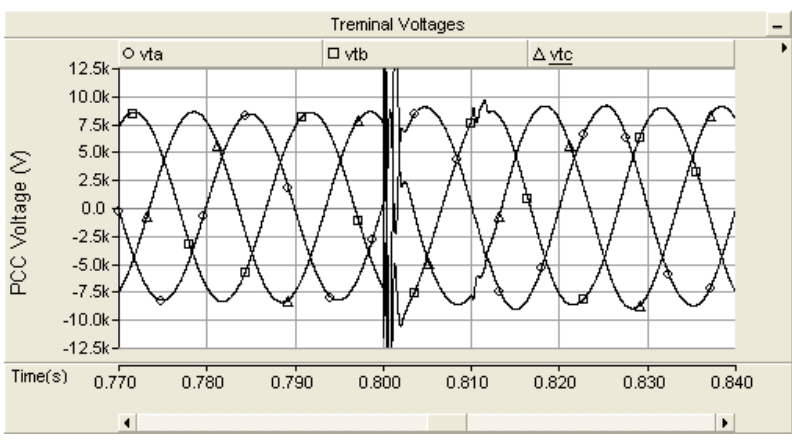

Fig. 6. PCC voltage before and after DSTATCOM connection (Example-1).

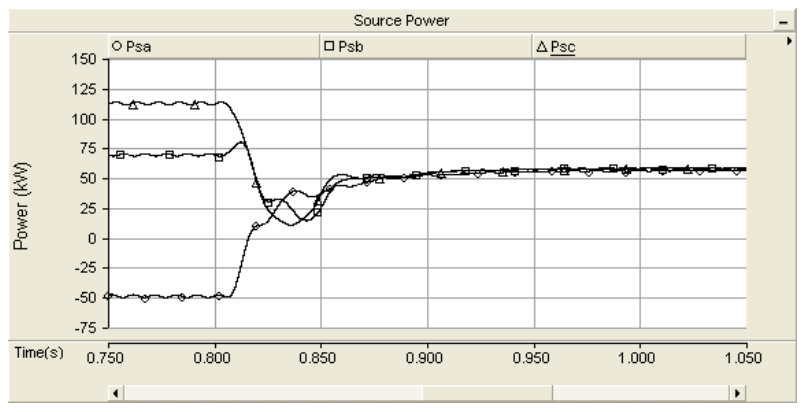

Fig. 7. Source power before and after DSTATCOM connection (Example-1). 


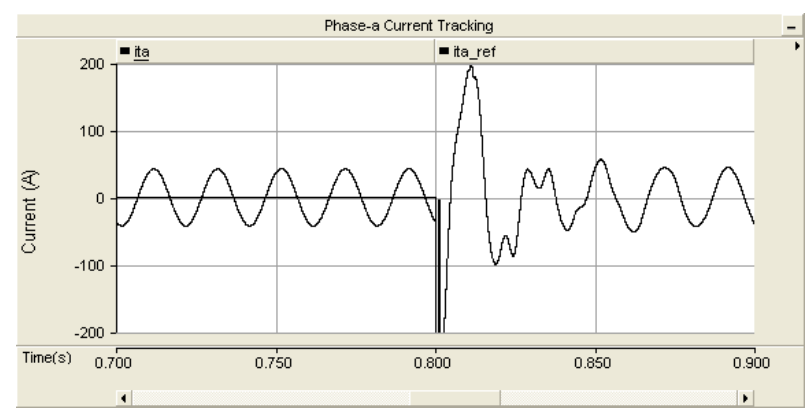

Fig. 8. Current tracking performance (Example-1).

Example 2: In this example, we consider the extension to the distribution system of Fig. 2 as shown in Fig. 9. In this, Feedeer-2 parameters are the same as $R_{s}$ and $L_{s}$ of Table I. Load-1 impedance is also same as given in Table I, whereas Load-2 impedances are $150+\mathrm{j} 235.62 \Omega, 200+\mathrm{j} 157.1 \Omega$ and $100+\mathrm{j} 157.1 \Omega$ in phases a, $\mathrm{b}$ and c respectively.

All the DERs inject a reactive power of $5 \mathrm{kVar}$. DER-1 is connected to phase-a at $0.2 \mathrm{~s}$ supply $300 \mathrm{~kW}$. DER-2 consisits of two separate DERs that are connected to phases b and c independently. Phase-b DER-2 is connected at $0.4 \mathrm{~s}$ supplying $150 \mathrm{~kW}$, while that is connected in phase-c at $0.5 \mathrm{~s}$ is supplying $250 \mathrm{~kW}$. The DSTATCOM is connected at $1.75 \mathrm{~s}$.

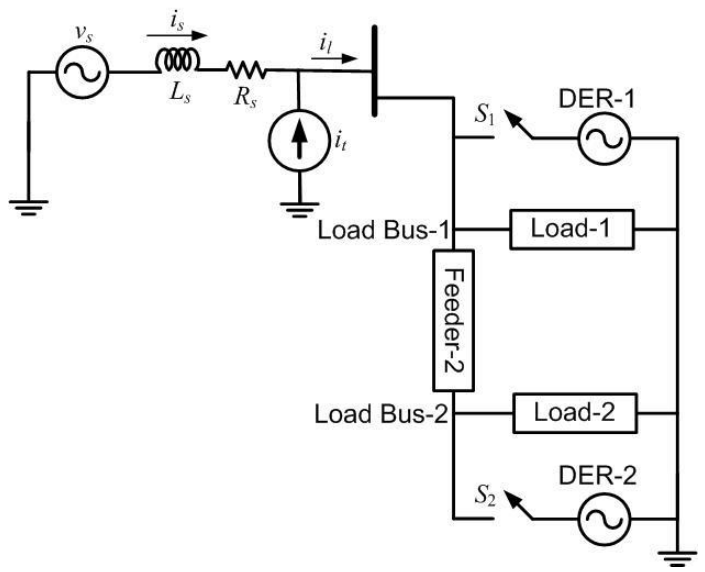

Fig. 9. A distribution system consisting of two load buses.

The power in the three phases is shown in Fig. 10. It can be seen that, before that DSTATCOM connection, power in two phases are negative. The power supplied by the phases becomes balanced once the DSTATCOM is connected, albeit the total power is negative. This implies that the DERs are supplying power to the source. To verify this point, the angle of the positive sequence of the PCC voltage is shown in Fig. 11. When no DER is connected, this angle is negative, indicating that power is flowing from the source to the load. Once the DERs start getting connected this angle becomes positive indicating a reverse power flow, however uncontrolled till the DSTATCOM gets connected. A scaled $\left(\times 10^{-3}\right)$ PCC phase-a voltage and current are shown in Fig. 12. It can be seen that they have a phase difference of $180^{\circ}$, which is implies that a unity power factor current is now flowing from the PCC to the source. The PCC voltage and source current in the steady state are shown in Figs. 13 and 14.

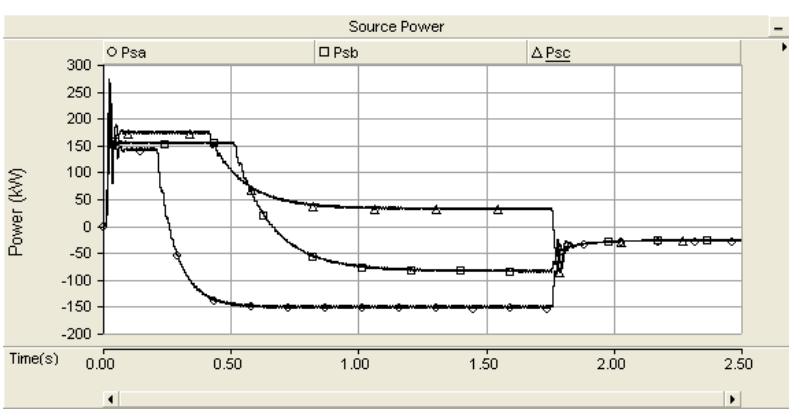

Fig. 10. Source power in the three phases (Example-2).

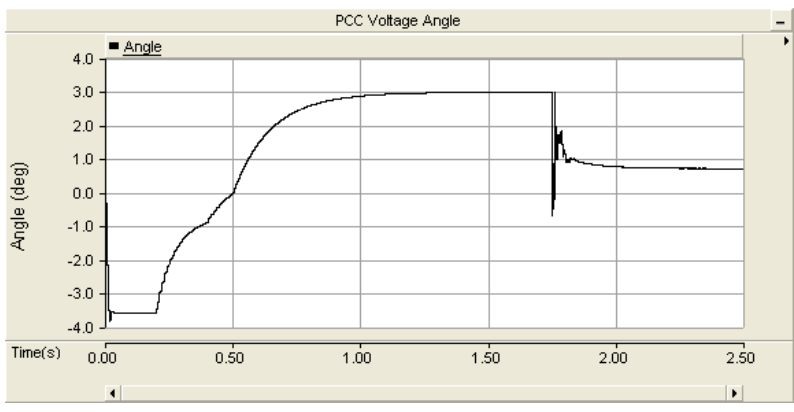

Fig. 11. Positive sequence PCC voltage angle (Example-2).

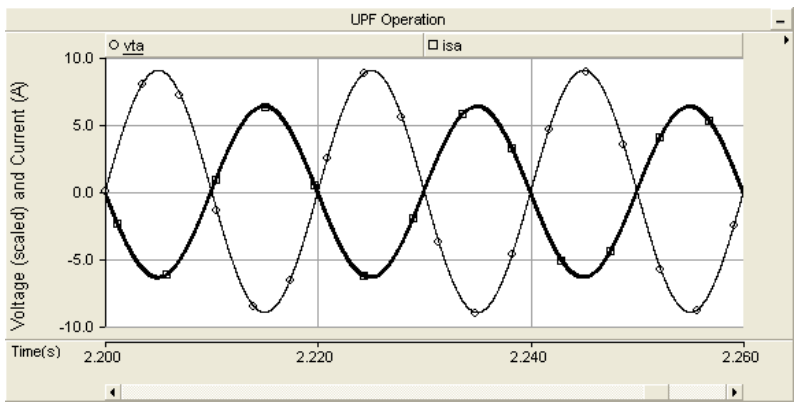

Fig. 12. PCC voltage and source current for UPF operation (Example-2).

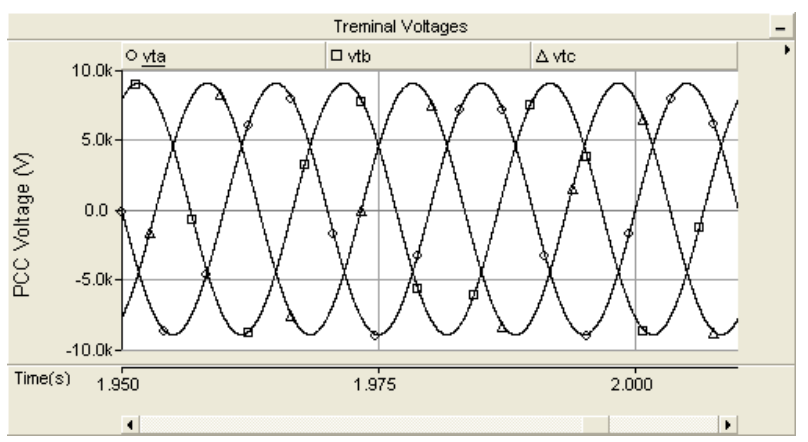

Fig. 13. Steady state PCC voltage (Example-2).

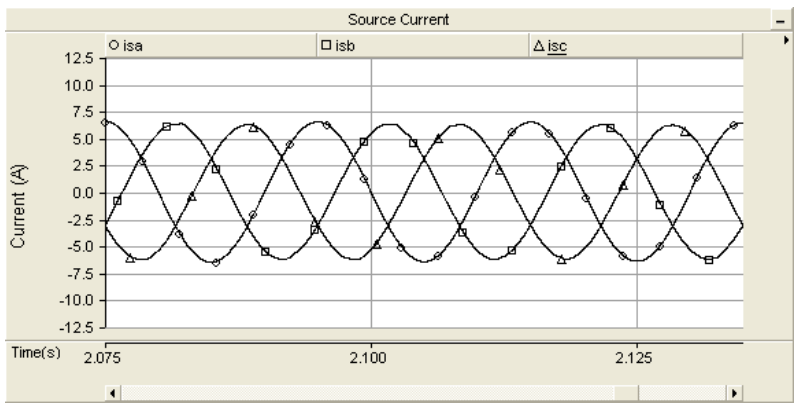

Fig. 14. Steady state source current (Example-2). 
Example 3: In this example, we consider the same system as given in Example 2, except that we connect the DSTATCOM to Load Bus-2 at $1.75 \mathrm{~s}$. The power supplied by the three phases of the source is shown in Fig. 15. It can be seen that the DSTATCOM cannot influence the power from DER-1 since it is connected downstream from Load Bus-1. Similarly, DSTATCOM cannot influence the phase-a source current, which has a negative polarity as shown in Fig. 16. The DSTATCOM however tries to balance the current upstream from Load Bus-2 and hence the current in phases b and c are balanced.

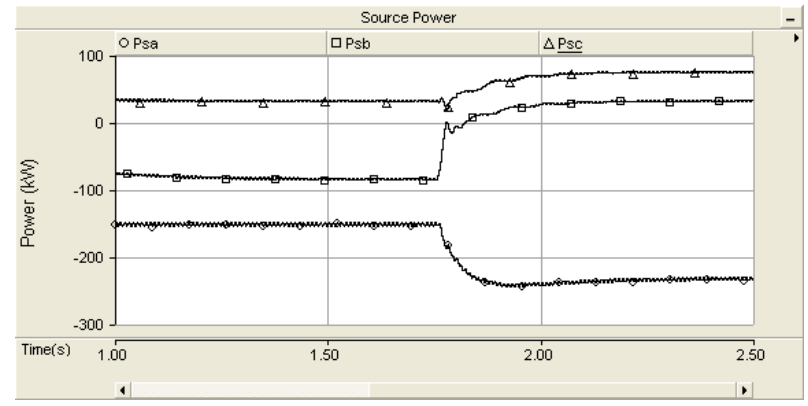

Fig. 15. Source power in the three phases (Example-3).

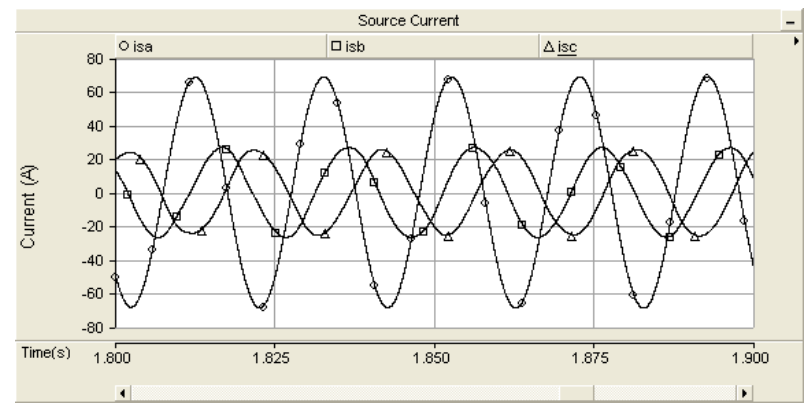

Fig. 16. Steady state source current (Example-3).

The above examples clearly demonstrate that a single DSTATCOM connected at the first bus itself can circulate power in all the three phases. However if the DSTATCOM is connected at any other bus, this will not be possible. It is to be noted that the DSTATCOM can only prevent unbalanced current from flowing in the upstream network and cannot influence the downstream current. In the next section, we shall discuss a strategy that can be employed if a particular threephase load amidst unbalanced loads and single-phase DERs required a set of balanced voltages.

\section{LOAD VOLTAGE CORRECTION}

So far only simple distribution systems have been considered. In this section, we shall consider a segment of a practical distribution system. This has 27 nodes as shown in Fig. 17. The system is connected with the substation through a $11 / 0.415 \mathrm{kV} \Delta$-Y transformer. As can be seen, the LV side of the system is not in pure radial nature. Every three-phase node is assumed to be consuming $6 \mathrm{~kW}$ power. However the loads at each node are assumed to be unbalanced. We have considered uniform length between two nodes and equal load at each node. The system data are shown in Table II. In addition 19 PV that are injecting power at unity power factor are connect- ed to the system - all in phase-a. Four of these PVs have a rating of $5 \mathrm{~kW}$ and the rest have a rating of $10 \mathrm{~kW}$.

TABLE-II: 27-NODE SYSTEM PARAMETERS

\begin{tabular}{|l|l|}
\hline \multicolumn{1}{|c|}{ System Quantities } & \multicolumn{1}{|c|}{ Values } \\
\hline Systems frequency & $50 \mathrm{~Hz}$ \\
\hline $\begin{array}{l}\text { Source (feeder) } \\
\text { impedance }\end{array}$ & $R_{s}=3.025 \Omega, L_{s}=57.8 \mathrm{mH}$ \\
\hline $\begin{array}{l}\text { Load impedance of } \\
\text { each node }\end{array}$ & $\begin{array}{l}\text { Phase-a: } 51.81+j 17.03 \Omega \\
\text { Phase-b: } 25.9+j 8.52 \Omega \\
\text { Phase-c: } 17.27+j 5.68 \Omega\end{array}$ \\
\hline $\begin{array}{l}\text { Impedance of line } \\
\text { segment between } \\
\text { nodes }\end{array}$ & $0.02+j 0.01 \Omega$ \\
\hline
\end{tabular}

We shall first investigate the voltage profile of the system before and after the PV connection. A load flow program is developed in MATLAB for the unbalanced network including the return neutral wire. The percentage voltage unbalance (VU) is then calculated from [15]

$$
V U=\left|\frac{V_{2}}{V_{1}}\right| \times 100 \%
$$

where $V_{2}$ and $V_{1}$ respectively are the negative and positive sequence rms voltage magnitudes. Voltage unbalance at each of the 27 nodes of the system is shown in Fig. 18. It can be seen that the unbalance increases significantly after the PV connections. It is not surprising that the voltage unbalance is higher in the nodes that are further from the substation (e.g. nodes 15 and 16).

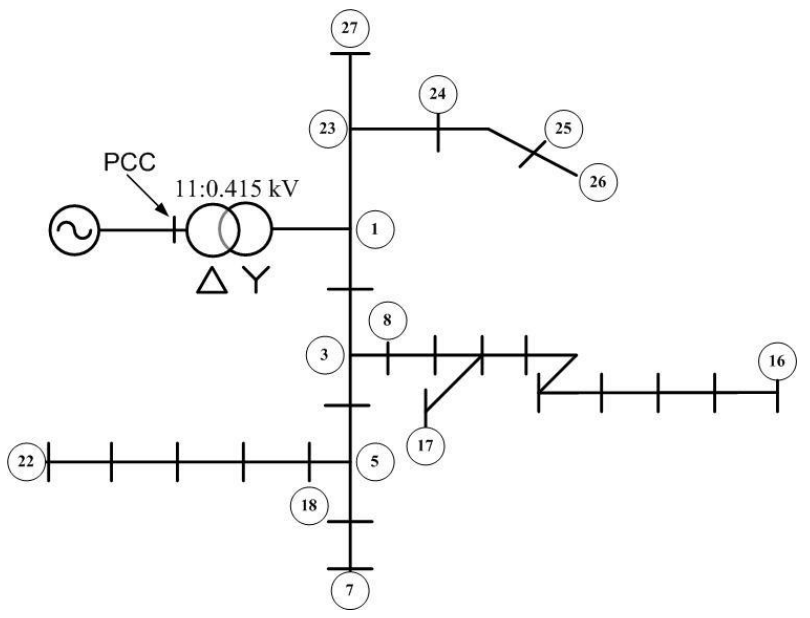

Fig. 17. A distribution system consisting of 27 nodes.

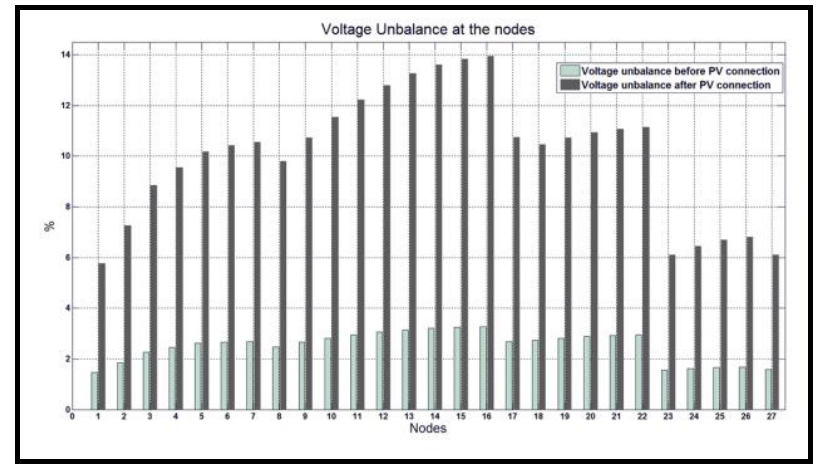

Fig. 18. Voltage unbalance profile along the LV network. 
A DSTATCOM is now connected at the MV side of the delta-wye transformer, at PCC. The rating of the DSTATCOM is the same as that given in Table I. In this case, the PVs are connected at $0.2 \mathrm{~s}$, while the DSTATCOM is connected at 0.5 s. The unbalance in PCC voltage is shown in Fig. 19. Before $\mathrm{PV}$ connection, the unbalance is less than $1 \%$, which reaches to $3.0 \%$ after PV connection. But after DSTATCOM connection it decreases rapidly to almost zero indicating that the DSTATCOM balances the PCC voltage.

The power supplied by each of the three phases of the source is shown in Fig. 20, while the source currents are shown in Fig. 21. It can be seen that the source power and the source currents almost become zero. Therefore the PVs support all the loads of the network.

However, the VU at the node furthest away from the substation (i.e., node-16), remains at a very high level despite the DSTATCOM connection (see Fig. 22). Usually the singlephase loads do not get affected by the voltage unbalanced in the LV feeders. However, if there are three-phase loads (e.g., elevators and pumps) amidst single-phase loads, large negative phase sequence (NPS) voltage can affect their performance. Let us assume that one such load is connected to node16. There are two possible solutions to this problem - the connection of a DVR at node-16 or a DSTATCOM connection between nodes 8 and 16. These are discussed below.

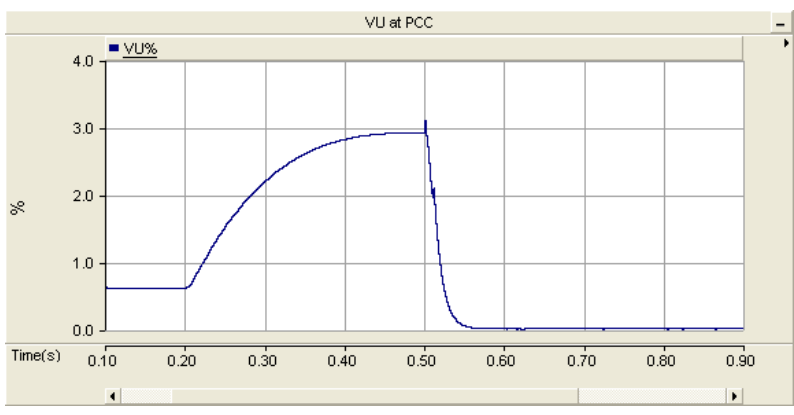

Fig. 19. VU at PCC before and after DSTATCOM connection.

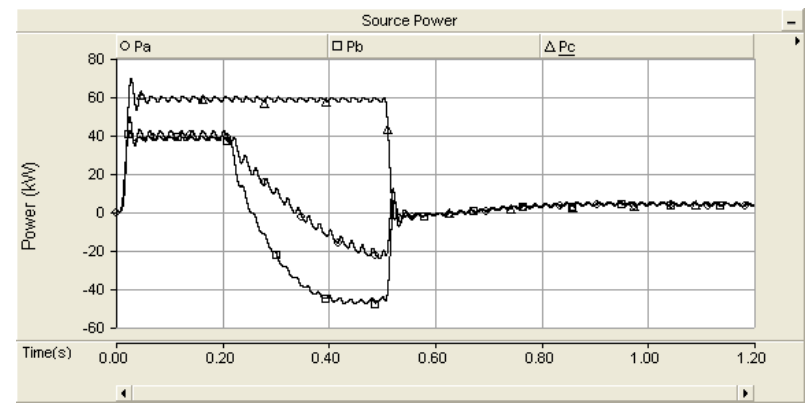

Fig. 20. Source power in the three phases.

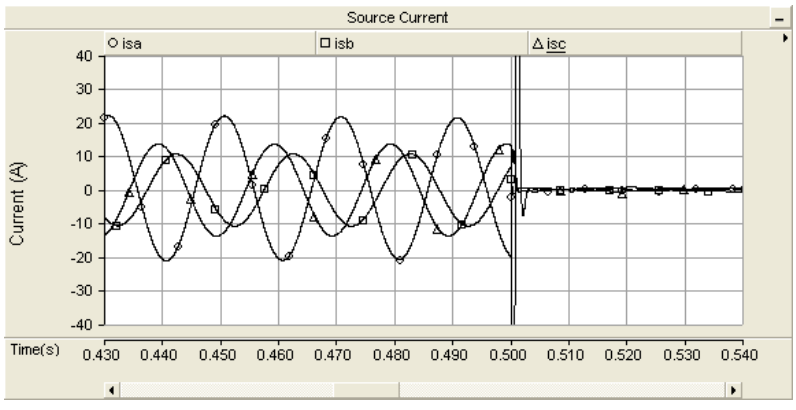

Fig. 21. Source current in the three phases.

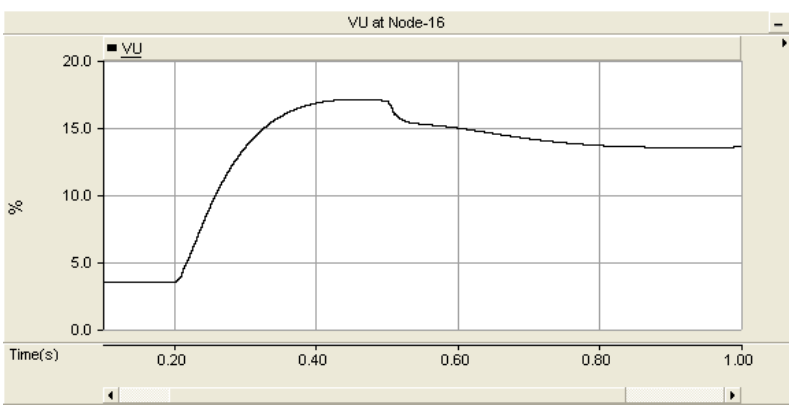

Fig. 22. VU at Node-16 before and after DSTATCOM connection.

\section{A. DVR Connection at Node-16}

A DVR can fix the voltage downstream and hence it can be connected between node-16 and its load, as shown in Fig. 23 (a). The DVR structure is essentially the same that shown in Fig. 1, except that the capacitor is connected on the secondary of the transformer and the voltage across the capacitor is controlled in the output feedback scheme discussed in Section II. The rating of the DVR is much smaller that the DSTATCOM given in Table I. Fig. 24 shows the VU across the load connected in node-16. The DVR is connected from the beginning, while the PVs and DSTATCOM are connected at $0.2 \mathrm{~s}$ and 0.5 $s$ respectively. It can be seen that the $\mathrm{VU}$ has been reduced to around $0.5 \%$. It is to be noted that the DVR injects a maximum peak voltage of $50 \mathrm{~V}$.

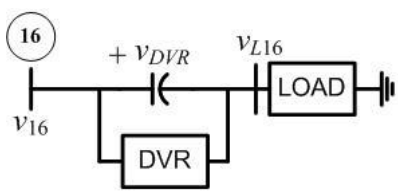

(a)

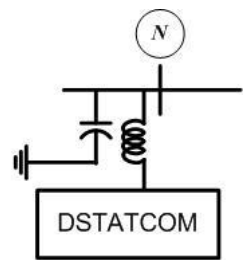

(b)
Fig. 23. (a) DVR connection for single load and (b) DSTATCOM connection at a bus.

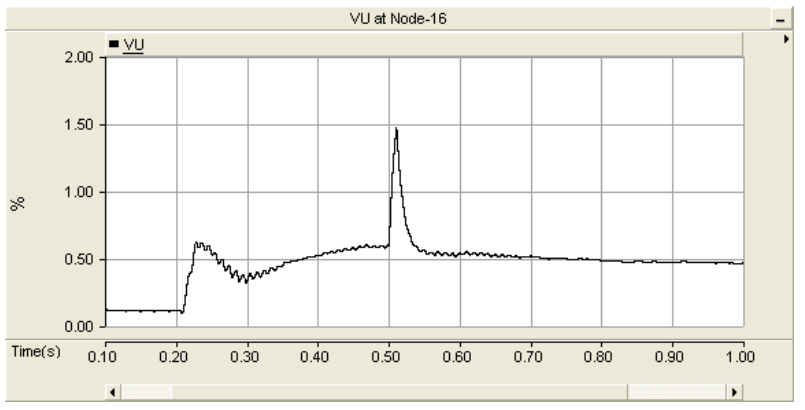

Fig. 24. VU at node-16 after DVR connection.

\section{A. DSTATCOM Connection at Node-14}

The DSTATCOM in this case has only an LC filter, where the filter capacitor is connected in the secondary side of the transformer in shunt with the node to which it is connected (see Fig. $23 \mathrm{~b}$ ). The rating of the DSTATCOM however is much smaller than that of the current balancing one. For this case, the DSTATCOM is connected at node-14. The voltage across filter capacitor is controlled in an output feedback voltage control. It has been assumed that the DSTATCOM remains connected to node-14 from the beginning. 
Since the DSTATCOM balances the voltage across node14 , it will also have a positive impact on the voltages of the neighboring nodes. Fig. 25 shows the $\mathrm{VU}$ in buses 11 to 16 with this DSTATCOM. The VU in all these nodes was more that $10 \%$ after PV connection, with or without the main (outer) DSTATCOM. It can be seen that this inner DSTATCOM reduces all the VUs to less than $3 \%$. Another important thing is to be noted that the VUs in the upstream nodes reduce after the outer DSTATCOM connection, while the VUs increase in node-14 and the downstream nodes. This is in agreement with what has been reported in [12].

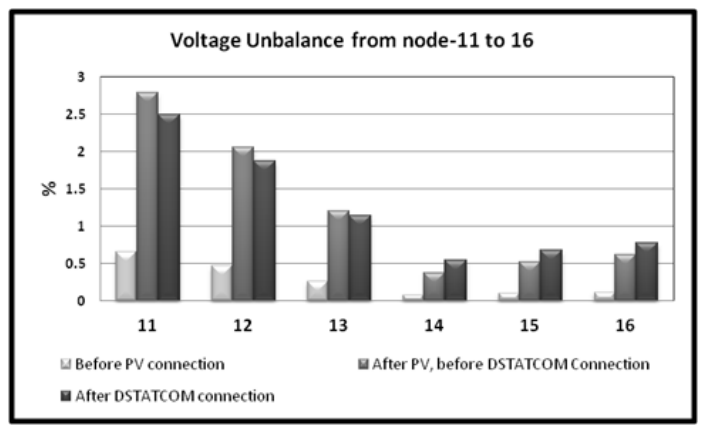

Fig. 25. VUs in nodes 11-16 after DSTATCOM connection.

\section{CONCLUSIONS}

In this paper, the power circulation capability of the DSTATCOM has been investigated. As it can be seen, if one phase in a system is generating more power than the others due to the existence of large number of single-phase DERs in that phase, the DSTATCOM can circulate the extra power from one phase to other phases to make them balance. The DSTATCOM can prevent the flow of large unbalanced current to the upstream MV network. It has been shown that the DSTATCOM facilitates the flow of balanced current to and from the upstream substation.

The main purpose of the DSTATCOM is to balance the current flowing from the substation and to distribute extra power generated amongst the phases. It however has no effect on the downstream distribution network. The downstream threephase loads can then be supplied through a customer installed DVR or a utility installed small rated DSTATCOM, both controlled to balance voltages.

In general most of the loads in the distribution systems are single-phase household loads. Therefore power imbalance does not affect them provided that the voltages are within a specified range. However a large injection in one phase can make the voltage in that phase to rise above acceptable limits. In such cases, the DSTATCOM connection is preferable since it can lower the voltages of a group of neighboring buses. Alternatively, the PVs can be controlled in a droop in which they can supply or absorb reactive power to prevent voltage rise or drop. This will be investigated in a separate study.

\section{REFERENCES}

[1] Y. Zhu and K. Tomsovic, "Adaptive power flow method for distribution systems with dispersed generation," IEEE Transactions on Power Delivery, vol. 17, pp. 822-827, 2002.
[2] T. E. McDermott and R. C. Dugan, "Distributed generation impact on reliability and power quality indices," IEEE Rural Electric Power Conference, pp. D3-D3_7, 2002.

[3] N. C. Scott, D. J. Atkinson, and J. E. Morrell, "Use of load control to regulate voltage on distribution networks with embedded generation," IEEE Transactions on Power Systems, vol. 17, pp. 510-515, 2002.

[4] R. A. Walling, R. Saint, R. C. Dugan, J. Burke, and L. A. Kojovic, "Summary of distributed resources impact on power delivery systems," IEEE Transactions on Power Delivery, vol. 23, pp. 1636-1644, 2008.

[5] A. Ghosh and G. Ledwich, Power Quality Enhancement using Custom Power Devices. Boston; Kluwer Academic, 2002.

[6] T. A. Short, Electric Power Distribution Handbook. Boca Raton, FL: CRC Press, 2004.

[7] A. Ghosh and A. Joshi, "A new approach to load balancing and power factor correction in power distribution system," IEEE Transactions on Power Delivery, vol. 15, pp. 417-422, 2000.

[8] C. Ben-Sheng and H. Yuan-Yih, "A Minimal Harmonic Controller for a STATCOM," IEEE Transactions on Industrial Electronics, vol. 55, pp. 655-664, 2008

[9] M. A. Eltawil and Z. M. Zhao, "Grid-connected photovoltaic power systems: Technical and potential problems-A review," Renewable \& Sustainable Energy Reviews, vol. 14, pp. 112-129, Jan 2010.

[10] J. A. P. Lopes, N. Hatziargyriou, J. Mutale, P. Djapic, and N. Jenkins, "Integrating distributed generation into electric power systems: A review of drivers, challenges and opportunities," Electric Power Systems Research, vol. 77, pp. 1189-1203, Jul 2007.

[11] S. A. Papathanassiou, "A technical evaluation framework for the connection of DG to the distribution network," Electric Power Systems Research, vol. 77, pp. 24-34, Jan 2007.

[12] F. Shahnia, A. Ghosh, G. Ledwich, and F. Zare, "Voltage Unbalance reduction in low voltage distribution networks with rooftop PVs," $20^{\text {th }}$ Australasian Universities Power Engineering Conference (AUPEC), 2010.

[13] A. Ghosh, A. K. Jindal, and A. Joshi, “"Inverter control using output feedback for power compensating devices," IEEE Asia-Pacific Region10 Conference (TENCON), vol. 1, pp. 48-52, 2003.

[14] A. Ghosh and G. Ledwich, "Load compensating DSTATCOM is weak ac systems," IEEE Transactions on Power Delivery, vol. 18, pp. 13021309, 2003.

[15] F. Shahnia, R. Majumder, A. Ghosh, G. Ledwich and F. Zare, "Voltage imbalance analysis in residential low voltage distribution networks with rooftop PVs," Electric Power Systems Research, vol. 81, pp. 18051814, 2011.

Sumit Mazumder (S'11) received his B.Sc. degree in Electrical Engineering from Bangladesh University of Engineering and Technology (BUET), Dhaka in 2009. From 2009-2011 he was employed in Robi Axiata Ltd., Dhaka, Bangladesh. Currently he is a Ph.D. student in Queensland University of Technology, Brisbane, Australia. His interests are in smart distribution grids and their power quality problems.

Arindam Ghosh (S'80, M'83, SM'93, F'06) is the Professor of Power Engineering at Queensland University of Technology, Brisbane, Australia. He has obtained a Ph.D. in EE from University of Calgary, Canada in 1983. Prior to joining the QUT in 2006, he was with the Department of Electrical Engineering at IIT Kanpur, India, for 21 years. He is a fellow of Indian National Academy of Engineering (INAE) and IEEE. His interests are in Control of Power Systems and Power Electronic devices.

Farhad Shahnia received his B.Sc. and M.Sc. in Electrical Power Engineering with first class honor from University of Tabriz, Tabriz, Iran in 2004 and 2006, respectively and his Ph.D. Electrical Engineering from Queensland University of Technology, Brisbane, Australia in 2011. His is currently a research fellow in Queensland University of Technology. His fields of interest include Distribution Networks, Power Quality, DG and Power Electronic Application in Power Systems.

Firuz Zare (M'97, SM'06) was born in Iran in 1967. He holds a PhD degree in Electrical Engineering from Queensland University of Technology in Australia. He has worked as a development engineer and a consultant in industry for several years. He has joined the school of engineering systems in QUT in 2006. His research interests are power electronic applications, pulse-width modulation techniques, renewable energy systems and electromagnetic interferences. 
Gerard Ledwich (M'73, SM'92) received the Ph.D. in electrical engineering from the University of Newcastle, Australia, in 1976. He has been Chair Professor in Power Engineering at Queensland University of Technology, Australia since 2006. Previously he was the Chair in Electrical Asset Management from 1998 to 2005 at the same university. He was Head of Electrical Engineering at the University of Newcastle from 1997 to 1998. Previously he was associated with the University of Queensland from 1976 to 1994 . His interests are in the areas of power systems, power electronics, and controls. He is a Fellow of I.E.Aust. 